\title{
Spinning particle interacting with electromagnetic and antisymmetric gauge fields in anti-de Sitter space
}

\author{
D. V. Uvarov ${ }^{\mathrm{a}}$ (i) \\ NSC Kharkov Institute of Physics and Technology, Kharkov 61108, Ukraine
}

Received: 7 November 2018 / Accepted: 12 May 2019 / Published online: 20 May 2019

(C) The Author(s) 2019

\begin{abstract}
Massless spinning particle model that interacts with electromagnetic and antisymmetric gauge fields in antide Sitter space-time is considered as a constrained Hamiltonian system. $d$-dimensional anti-de Sitter space-time is realized as a real projective manifold parametrized by the homogeneous coordinates. Classical constraints that generate in the presence of interactions minimal world-line supersymmetry algebra extended by the dilatations of the ambientspace homogeneous coordinates are found. Various representations of the Lagrangian of the spinning particle are obtained. Dirac quantization is shown to produce first- and second-order equations for the wave function of the spinning particle that are presented in the homogeneous, inhomogeneous and intrinsic coordinates of $A d S_{d}$.
\end{abstract}

\section{Introduction}

Spinning particle models [1-4] are known to provide classical realization of the spin 1/2 field equations in Minkowski space-time as odd generators of the minimal world-line supersymmetry algebra that is the finite-dimensional subalgebra of the infinite-dimensional superVirasoro algebra of the superstring. Since the world-line supersymmetry is less restrictive than the world-sheet one, spinning particle models admit a wide variety of generalizations. In particular, it is possible to include interactions with background electromagnetic [1,5-8], Yang-Mills [9,10], gravitational [5,11] and antisymmetric gauge fields [12] in a way consistent with minimal world-line supersymmetry. Such models upon quantization yield Dirac equation for spin 1/2 field interacting with background fields. Apart from Minkowski space-time of the special interest are maximally symmetric spaces such as anti-de Sitter space-time. There the interplay between the space-time geometry and world-line supersymmetry appears to be quite non-trivial $[13,14]$.

\footnotetext{
a e-mail: d_uvarov@hotmail.com
}

Anti-de Sitter space can be described as a manifold embedded into flat space-time with extra dimension(s) and it is possible to consider respective spinning particle models [1518]. These provide pseudoclassical realization of the idea of formulating field dynamics in (anti-)de Sitter (as well as Minkowski) space in a way exhibiting (conformal) isometries that dates back to the seminal works of Dirac $[19,20]$. It was also applied to examine conformal field theories in 4-dimensional Minkowski (Euclidean) space-time [21-23] and to formulate dynamical equations for the gauge fields in 4-dimensional anti-de Sitter space [24,25]. More recently embedding (or ambient) space description was applied to study correlation functions in $d$-dimensional conformal field theories taking advantage of the AdS/CFT inspired techniques [26-28] and to study higher-spin field equations in $A d S_{d}$ and its conformal boundary [29-31]. In Ref. [32] there was considered the possibility of applying twistor methods to the $A d S / C F T$ duality based on the projective-space description of the bulk anti-de Sitter space parametrized by the homogeneous coordinates that naturally combines linear realization of $S O(2, d-1)$ isometry and the projective light-cone description of the $(d-1)$-dimensional conformal boundary space-time. Shortly after that two-twistor formulation of the spinning particle in $A d S_{d}$ for $d=4,5,7$ was proposed in [33]. It is based on the generalization [34] of the two-twistor formulation of the massive bosonic particle in $\operatorname{Ad} S_{5}[35]{ }^{1}$

Utility of the projective-space realization of anti-de Sitter space from the viewpoint of canonical description of massless particle (tensionless string) models can be justi-

\footnotetext{
${ }^{1}$ In $[33,34]$ there was also proposed two-supertwistor formulation of the particle model in $\operatorname{Ad} S_{d}(d=4,5,7)$ foliated by the superMinkowski slices. For $d=5$ complete twistor form of the massless superparticle action in (10|32)-dimensional $A d S_{5} \times S^{5}$ superspace was found in [36]. In addition to the pair of $S U(2,2 \mid 4)$ Penrose-Ferber supertwistors [37], it invloves the pair of $S U(2,2 \mid 4)$ supertwistors with the odd $S U(2,2)$ and even $S U(4)$ components in accordance with the earlier results of Ref. [38,39].
} 
fied as follows. Description of $A d S_{d}$ as an embedded hyperboloid assumes imposition of the constraint $y^{2}+1 \approx$ 0 on the ambient-space inhomogeneous coordinates $y \underline{m}$, where the $S O(2, d-1)$-invariant scalar product $y^{2}=$ $(y \cdot y)=y^{\underline{m}} \eta_{\underline{m n}} y^{\underline{n}}$ is taken w.r.t. Minkowski metric $\eta_{m n}=$ $\operatorname{diag}(-,-,+, \cdots,+)$ and $A d S_{d}$ radius is set to unity. In the canonical approach the mass-shell constraint for the massless particle (tensionless string zero modes) in its simplest form is $p^{2} \approx 0$ and its Poisson bracket $(\mathrm{PB})$ relations with the above constraint imply that $(y \cdot p) \approx 0$ is also a constraint forming with $y^{2}+1 \approx 0$ the pair of the second-class constraints. The presence of the second-class constraints necessitates introduction of the Dirac brackets (DB) that in general essentially complicates analysis of the Hamiltonian dynamics (see, e.g. [40]) so it is convenient to treat the constraint $y^{2}+1 \approx 0$ as a gauge-fixing condition for the first-class constraint $(y \cdot p) \approx 0$ that generates dilatations of the embeddingspace coordinates [41]. Gauged dilatations implement the projective-space realization of $A d S_{d}$, so the set of the two first-class constraints $(y \cdot p) \approx 0$ and $p^{2} \approx 0$ can be taken as the starting point for description of the massless particle (tensionless string zero modes) models in such an approach.

In the Lagrangian approach important feature of the parametrization of $A d S_{d}$ by the homogeneous coordinates $x^{\underline{m}}: y^{\underline{m}}=|x|^{-1} x^{\underline{m}},|x|=\sqrt{-x^{2}}$, is that the object that can be naturally identified with the metric tensor, taking into account the form of the line element,

$d s^{2}=\frac{1}{|x|^{2}} d x^{\underline{m}} \theta_{\underline{m n}} d x^{\underline{n}}, \quad \theta_{\underline{m n}}=\eta_{\underline{m n}}+\frac{1}{|x|^{2}} x_{\underline{m}} x_{\underline{n}}$,

is degenerate $\operatorname{det} \theta=0$. So one is led to consider particle (string, brane) mechanics in the space with degenerate metric [42]. Tensor $\theta_{\underline{m} n}$ and associated differential operator $\theta \frac{m n}{n} \partial / \partial x^{n}$ also enter dynamical equations for the $A d S_{d}$ higher-spin fields in the ambient-space formulation [24,25,43-45].

In Ref. [46] there was proposed massless spinning particle model in $A d S_{d}$ realized as the projective space parametrized by the homogeneous coordinates. Three first-class constraints of the model (one odd and two even) span minimal world-line supersymmetry algebra extended by the gauged space-time dilatations. Dirac quantization of the model yields Dirac and Klein-Gordon equations for the particle's wave function that is a homogeneous function of degree zero.

In this note we continue to study the above model and examine the possibility of including interactions with background gauge fields. As the starting point we take Hamiltonian first-class constraints of the free spinning particle model. Then we seek for the generalizations of the odd constraint, that is the world-line supersymmetry generator, by the terms depending on the background gauge fields and calculate its DB relations with itself that define bosonic con- straint generating world-line reparametrizations. Then linear combination of these constraints and the generator of the space-time dilatations with the Lagrange multipliers is used to write down the Lagrangian of the interacting spinning particle model in terms of the phase-space variables. These Lagrange multipliers play the role of the gauge fields for local world-line supersymmetry, reparametrizations and space-time dilatations. Integrating out space-time momentum and some of the Lagrange multipliers we derive various representations of the spinning particle Lagrangian. After that we discuss Dirac quantization of the proposed models. We find Hermitian operators associated with the classical first-class constraints from the requirement that they satisfy quantum world-line supersymmetry algebra. Then the substitution of the realization of the Hermitian momentum operator as a differential operator in configuration space produces Dirac- and Klein-Gordon-type equations for the wave function of the spinning particle in homogeneous coordinates. We also write these equations in the inhomogeneous and intrinsic coordinates on $A d S_{d}$.

Section 2 is devoted to the spinning particle's interaction with the background electromagnetic field. ${ }^{2}$ In Sect. 3 we discuss gauge-invariant interaction with the rank $r-1$ antisymmetric gauge field. Like in the case of electromagnetic interaction closed algebra of the constraints is obtained and various forms of Dirac- and Klein-Gordon-type equations for the particle's wave function are found. Let us remark that the spinning particle model with minimal world-line supersymmetry interacting with odd-rank antisymmetric tensor gauge fields in $(2 d+1)$-dimensional Minkowski space was studied in [12] from the perspective of the Kaluza-Klein dimensional reduction. In $2 d$ dimensions it results in the particle's interactions with both rank $2 r$ and $2 r+1$ antisymmetric gauge fields as well as with the electromagnetic field. Curiously antisymmetric gauge fields appear in quantization of the spinning particle model with extended world-line supersymmetry [53].

\section{Charged spinning particle in background electromagnetic field}

Consider odd constraint

$\Phi_{(e)}=|x| \xi \cdot(p-e A(x)) \approx 0$

as the classical analogue of the Dirac equation that includes interaction with external electromagnetic field. We take it as the generator of the minimal world-line supersymmetry.

\footnotetext{
2 (Non-minimal) electromagnetic interactions of the superparticle were considered in [47-52].
} 
In the absence of the interaction it coincides with odd constraint introduced in [46]. Observe that the minimality principle fixes the homogeneity degree of $A_{\underline{m}}(x):(x \cdot \partial) A(x)=$ $-A(x)$. Transverse strength of the electromagnetic field by definition is

$$
\begin{aligned}
& F_{\underline{m n}}(x)=\theta_{\underline{m}} \underline{\underline{k}} \theta_{\underline{\underline{n}}-}-\left(\partial_{\underline{k}} A_{\underline{l}}-\partial_{\underline{l}} A_{\underline{k}}\right)=\partial_{\underline{m}} A_{\underline{n}}-\partial_{\underline{n}} A_{\underline{m}}, \\
& \theta_{\underline{m}} \underline{\underline{n}}(x)=\delta_{\underline{m}} \underline{n}+\frac{x_{\underline{m}} x \underline{\underline{n}}}{|x|^{2}}
\end{aligned}
$$

and the last equality follows by taking into account transversality $(x \cdot A(x))=0$ and homogeneity properties of the electromagnetic potential. After introduction of the PB (DB) relations

$\left\{p_{\underline{m}}, x^{\underline{\underline{n}}}\right\}_{\mathrm{PB}}=\delta_{\underline{\underline{m}}}^{\underline{n}}, \quad\left\{\xi^{\underline{m}}, \xi^{\underline{n}}\right\}_{\mathrm{DB}}=i \eta^{\underline{m n}}$

it is easy to see that odd constraint (2) has zero PB with the constraint

$D=(x \cdot p) \approx 0$

that generates dilatations of the embedding-space coordinates. Also the DB relations of the supersymmetry generator with itself

$\left\{\Phi_{(e)}, \Phi_{(e)}\right\}_{\mathrm{DB}}=i T_{(e)}$

define bosonic constraint

$T_{(e)}=|x|(p-e A)^{2}+2 i(\xi \cdot x) \xi \cdot(p-e A)+i e|x|^{2}(\xi \cdot F \cdot \xi) \approx 0$

that is the generator of the world-line reparametrizations. Eq. (6) appears to be the only non-trivial relation of the world-line supersymmetry algebra extended by the spacetime dilatations.

Having introduced the classical first-class constraints we can write down the spinning particle's Hamiltonian as their linear combination

$\mathscr{H}_{(e)}=\frac{\tilde{e}}{2} T_{(e)}-a D-i \chi \Phi_{(e)} \approx 0$

with even $\tilde{e}, a$ and odd $\chi$ Lagrange multipliers. Then the action is defined as the integral of the Lagrangian expressed in terms of the phase-space variables

$S_{(e)}=\int d \tau \mathscr{L}_{(e) \mathrm{ph}}$,

where

$$
\mathscr{L}_{(e) \mathrm{ph}}=(p \cdot \dot{x})+\frac{i}{2}(\xi \cdot \dot{\xi})-\mathscr{H}_{(e)} .
$$

Integrating out the momentum $p_{m}$ yields configuration-space form of the particle's Lagrangian

$$
\begin{aligned}
\mathscr{L}_{(e) \text { conf }}= & \frac{1}{2 \tilde{e}|x|^{2}}(\dot{x}+a x)^{2}+e(\dot{x} \cdot A)+\frac{i}{2}(\xi \cdot \dot{\xi}) \\
& -\frac{i}{|x|^{2}}(\xi \cdot x)(\xi \cdot \dot{x}) \\
& +\frac{i x}{\tilde{e}|x|} \xi \cdot(\dot{x}+a x)-\frac{i e}{2} \tilde{e}|x|^{2}(\xi \cdot F \cdot \xi) .
\end{aligned}
$$

The Lagrange multiplier $a$ plays the role of the gauge field for the scale transformations of $x$ and $p$. Integrating it out allows to bring the Lagrangian to the form

$$
\begin{aligned}
\mathscr{L}_{(e) R P^{d}=} & \frac{1}{2 \tilde{e}|x|^{2}}(\dot{x} \theta \dot{x})+e(\dot{x} \cdot A)+\frac{i}{2}(\xi \cdot \dot{\xi}) \\
& -\frac{i}{|x|^{2}}(\xi \cdot x)(\xi \cdot \dot{x}) \\
& +\frac{i \chi}{\tilde{e}|x|}(\xi \theta \dot{x})-\frac{i e}{2} \tilde{e}|x|^{2}(\xi \cdot F \cdot \xi)
\end{aligned}
$$

that manifests the realization of $A d S_{d}$ as the projective space $R P^{d}$ parametrized by the homogeneous coordinates with the degenerate metric $\theta_{\underline{m n}}=\eta_{\underline{m n}}+\frac{1}{|x|^{2}} x_{\underline{m}} x_{\underline{n}}$.

In quantum theory classical observables are replaced by the Hermitian operators and their PB (DB) relations-by the (anti)commutators. The operators associated with the phasespace variables satisfy the (anti)commutation relations ${ }^{3}$

$\left[p_{\underline{m}}, x^{\underline{n}}\right]=-i \delta_{\underline{m}}^{\underline{n}}, \quad\left\{\xi^{\underline{m}}, \xi^{\underline{n}}\right\}=\eta^{\underline{m} n}$.

From the anticommutation relations of $\xi^{\underline{m}}$ it follows that they are proportional to $\gamma$-matrices in $(d+1)$ dimensions: $\xi^{\underline{m}}=2^{-1 / 2} \gamma^{\underline{m}}$ and their Hermiticity is understood in the same sense as that of $\gamma^{\underline{m}}$, i.e. $\left(\gamma^{\underline{m}}\right)^{\dagger}=(-)^{t} A \gamma^{\underline{m}} A^{-1}$, where $A=\gamma^{0_{1}} \gamma^{0_{2}} \cdots \gamma^{0_{t}}$ and $t$ is the number of time-like dimensions ( $t=2$ for the realization of $A d S_{d}$ as the hyperboloid in the ambient space-time). Classical constraints become Hermitian operators that select physical subspace in the space of quantum states of the spinning particle. We choose Hermitian operator associated with the classical supersymmetry generator in the form

$\Phi_{(e) \mathrm{H}}=|x| \gamma \cdot(p-e A)+\frac{i(\gamma \cdot x)}{2|x|} \approx 0$,

where the second summand arises as a result of moving the momentum operator to the right in the manifestly Hermitian representation for the first summand. The square of $\Phi_{(e) \mathrm{H}}$

$\Phi_{(e) \mathrm{H}}^{2}=T_{(e) \mathrm{H}}$

\footnotetext{
3 We do not place hats over the quantum operators and set $\hbar=1$ not to overburden the notation. Hopefully, this will not cause a confusion.
} 
defines Hermitian operator associated with the classical constraint $T_{(e)}{ }^{4}$

$T_{(e) \mathrm{H}}=[|x| \gamma \cdot(p-e A)]^{2}+i D_{\mathrm{H}}-\frac{1}{4} \approx 0$,

where

$D_{\mathrm{H}}=(x \cdot p)-\frac{i(d+1)}{2} \approx 0$

is the Hermitian operator for the generator of the space-time dilatations. Note the relation

$$
\begin{aligned}
{[|x| \gamma \cdot(p-e A)]^{2}=} & |x|^{2}(p-e A)^{2}+i(\gamma \cdot x) \gamma \cdot(p-e A) \\
& +\frac{i e}{2}|x|^{2}(\gamma \cdot F \cdot \gamma)
\end{aligned}
$$

that makes obvious the contact with the classical constraint (7).

For the case of flat configuration-space Hermitian momentum operator can be realized as the coordinate partial derivative acting on the wave function $\Psi(x)$. Whenever configuration space is a curved manifold, Hermitian momentum operator is given by

$p_{\underline{m}}=-i(-g)^{-\frac{1}{4}} \partial_{\underline{m}}(-g)^{\frac{1}{4}}$,

where $g$ is the determinant of the configuration-space metric tensor. In the realization of anti-de Sitter space-time as the projective manifold, the scale-invariant measure is proportional to $|x|^{-d-1} \varepsilon_{\underline{m}_{1}} \underline{m}_{2} \cdots \underline{m}_{d+1} x^{\underline{m}_{1}} d x^{\underline{m}_{2}} \wedge \cdots \wedge d x^{\underline{m}} d+1$, so as the definition of the Hermitian momentum operator we take

$p_{\underline{m}}=-i|x|^{\frac{d+1}{2}} \partial_{\underline{m}}|x|^{-\frac{d+1}{2}}=-i\left(\partial_{\underline{m}}+\frac{(d+1)}{2|x|^{2}} x_{\underline{m}}\right)$.

Then the constraint (14) translates into the Dirac-type equation

$\Phi_{(e) \mathrm{H}} \Psi(x)=-i|x| \gamma \cdot(\partial-i e A) \Psi(x)-\frac{i d(\gamma \cdot x)}{2|x|} \Psi(x)=0$

for the particle's wave function $\Psi(x)$ that is the $2^{\left[\frac{d+1}{2}\right]}$. component spinor field. It has the homogeneity degree zero $D_{\mathrm{H}} \Psi(x)=(x \cdot \partial) \Psi=0$ and also satisfies the second-order equation

$T_{(e) \mathrm{H}} \Psi(x)=-[|x| \gamma \cdot(\partial-i e A)]^{2} \Psi(x)-\frac{d^{2}}{4} \Psi(x)=0$.

\footnotetext{
${ }^{4}$ Discussion of the ambiguities in the definition of Hermitian operators in locally supersymmetric models can be found, e.g., in [54-56].
}

To conclude this section let us discuss how the conventional form of spin 1/2 particle's equations in $A d S_{d}$ in terms of intrinsic coordinates can be derived from the equations given above. As an intermediate step let us present Eqs. (21) and (22) in the inhomogeneous coordinates $y^{\underline{m}}=|x|^{-1} x^{\underline{m}}$. For Eq. (21) we obtain

$$
\Phi_{(e) \mathrm{H}} \Psi(y)=-i \gamma \cdot(\nabla-i e A) \Psi(y)-\frac{i d}{2}(\gamma \cdot y) \Psi(y)=0,
$$

where $\nabla_{\underline{m}}=\theta_{\underline{m}} \underline{\underline{n}}(y) \partial / \partial y^{\underline{n}}, \theta_{\underline{m}}^{\underline{n}}(y)=\delta_{\underline{m}}^{\underline{n}}+y_{\underline{m}} y^{\underline{n}}$, and Eq. (22) becomes

$T_{(e) \mathrm{H}} \Psi(y)=-[\gamma \cdot(\nabla-i e A)]^{2} \Psi(y)-\frac{d^{2}}{4} \Psi(y)=0$.

The electromagnetic potential and field strength in the homogeneous and inhomogeneous coordinates are related as

$|x| A_{\underline{m}}(x)=A_{\underline{m}}(y), \quad|x|^{2} F_{\underline{m n}}(x)=F_{\underline{m n}}(y)$.

Transverse field strength in the inhomogeneous coordinates is defined by

$F_{\underline{m n}}(y)=\theta_{\underline{m}} \underline{\underline{k}} \theta_{\underline{n}} \underline{l}\left(\partial_{\underline{k}} A_{\underline{l}}-\partial_{\underline{l}} A_{\underline{k}}\right)=\left(\partial_{\underline{m}}+y_{\underline{m}}\right) A_{\underline{n}}-\left(\partial_{\underline{n}}+y_{\underline{n}}\right) A_{\underline{m}}$

and the last equality follows by using the transversality property of the potential $y \cdot A(y)=0$.

Above equations for the particle's wave function in the inhomogeneous coordinates can be transformed to intrinsic coordinates using the transition formulae $[24,25,45]$. In particular, we use the relation between the derivatives of the coordinate functions

$\nabla^{\underline{m}} z^{m}=g^{m n} \partial_{n} y^{\underline{m}}$,

where $g_{m n}(z)=\partial_{m} y \underline{\underline{m}} \partial_{n} y_{\underline{m}}$ and $g^{m n}(z)=\nabla \underline{m} z^{m} \nabla_{\underline{m}} z^{n}$ are the $A d S_{d}$ metric and its inverse in the intrinsic coordinates. Also the spinning particle's wave functions in the inhomogeneous and intrinsic coordinates are connected by the $2^{\left[\frac{d+1}{2}\right]} \times 2^{\left[\frac{d+1}{2}\right]}$ matrix $M(z)$

$\Psi(y)=M \psi(z):$

$M^{-1} \partial_{m} M=\frac{1}{2} \omega_{m}{ }^{a b} \sigma_{a b}+\frac{1}{2} e_{m}^{a} \rho_{a}$,

$M^{-1}(\gamma \cdot y)\left(\gamma \cdot \partial_{m} y\right) M=e_{m}^{a} \rho_{a}$.

In Eqs. (29) and (30) $e_{m}^{a}(z)$ and $\omega_{m}{ }^{a b}(z)$ are the $A d S_{d}$ vielbein and spin connection. Eq. (29) implies that $M \in$ 
$S O(2, d-1) / S O(1, d-1)$ with the $s o(2, d-1)$ generators realized by the Dirac matrices in $d$ dimensions $\rho^{a}$ :

$\rho^{a} \rho^{b}+\rho^{b} \rho^{a}=2 \eta^{a b}$

and $\sigma_{a b}=\frac{1}{4}\left(\rho_{a} \rho_{b}-\rho_{b} \rho_{a}\right)$ that span the $s o(1, d-1)$ algebra. Useful consequences of Eqs. (29) and (30) are

$\left(\gamma \cdot \partial_{m} y\right)=M \rho_{m} M^{-1}(\gamma \cdot y), \quad\left\{M \rho_{m} M^{-1},(\gamma \cdot y)\right\}=0$,

$\rho_{m}=e_{m}^{a} \rho_{a}$.

To obtain conventional form of the Dirac equation we multiply Eq. (23) by $M^{-1}(\gamma \cdot y)$ from the left. Application of the transition relations (27)-(30) allows to obtain massless Dirac equation in $A d S_{d}$

$i M^{-1}(\gamma \cdot y) \Phi_{(e) \mathrm{H}}(y) M \psi(z)=\rho^{m} D_{m}(A) \psi(z)=0$,

where

$D_{m}(A)=\partial_{m}+\frac{1}{2} \omega_{m}^{a b} \sigma_{a b}-i e A_{m}$

is the spinor covariant derivative extended by the external electromagnetic potential. The commutator of the covariant derivatives

$\left[D_{a}(A), D_{b}(A)\right]=\frac{1}{2} R_{a b}{ }^{c d} \sigma_{c d}-i e F_{a b}=-\sigma_{a b}-i e F_{a b}$,

$D_{a}(A)=e_{a}^{m} D_{m}(A)$

appears in the transformation of the second-order equation (24) to the intrinsic coordinates. To find the final form we substituted explicit expression for the Riemann tensor

$R_{k l m n}=g_{k n} g_{l m}-g_{k m} g_{l n}, \quad\left(R=R_{m n}^{m n}=-d(d-1)\right)$

that provides solution of the Einstein equations in the form widely used in the literature on the $A d S / C F T$ correspondence:

$R_{m n}-\frac{1}{2} g_{m n} R+\Lambda g_{m n}=0$

with the cosmological constant $\Lambda=-\frac{(d-1)(d-2)}{2}$. The resulting Klein-Gordon-type equation is

$$
\begin{aligned}
-M^{-1} T_{(e) \mathrm{H}} M \psi(z)= & D^{2}(A) \psi-i e \sigma^{m n} F_{m n} \psi \\
& +\frac{d(d-1)}{4} \psi=0 .
\end{aligned}
$$

Note that $\left[(\gamma \cdot y) \Phi_{(e) \mathrm{H}}\right]^{2}$ differs from $\Phi_{(e) \mathrm{H}}^{2}$ by the linear combination of the constraints $\Phi_{(e) \mathrm{H}}$ and $D_{\mathrm{H}}$.

\section{Spinning particle interactions with antisymmetric gauge fields}

In this section we discuss gauge-invariant coupling of the spinning particle to external $(r-1)$-form gauge field $A_{\underline{m}[r-1]}(x)^{5}$ that we assume to be transverse $x^{\underline{n}} A_{\underline{n} \underline{[}[r-2]}(x)$ $=0$ and homogeneous of degree $-(r-1)$. The definition of the transverse field strength

$F_{\underline{m}[r]}(x)=\theta_{\underline{m}_{1}}{ }^{\underline{n}_{1}} \theta_{\underline{m}_{2}} \underline{\underline{n}}_{2} \ldots \theta_{\underline{m}_{r}} \underline{n}_{r} \partial_{\left[\underline{n}_{1}\right.} A_{\left.\underline{n}_{2} \ldots \underline{n}_{r}\right]}=\partial_{\left[\underline{m}_{1}\right.} A_{\left.\underline{m}_{2} \ldots \underline{m}_{r}\right]}$

generalizes that for the electromagnetic field (3). Since the form of the coupling is sensitive to the value of $r$ we start with the case of odd $r$ and then turn to even $r$.

\section{$3.1 r$ odd}

In this case the fermionic constraint

$\Phi_{(q, r \text { odd })}=|x|(\xi \cdot p)+i^{n} q|x|^{r} \xi^{\underline{\underline{m}}[r]} F_{\underline{m}[r]} \approx 0$

naturally generalizes that for the free spinning particle. $q$ stands for the particle's charge, $\xi^{\underline{m}[r]}=\xi^{\underline{m_{1}}} \ldots \xi^{\underline{m_{r}}}$ and the factor $|x|^{r}$ makes the last term homogeneous of degree zero like the first is, while the factor $i^{n}, n=\frac{r-1}{2}-2\left[\frac{r-1}{4}\right]$ makes it real under the complex conjugation. DB relations of this constraint with itself generate classical world-line supersymmetry algebra

$\left\{\Phi_{(q, r \text { odd })}, \Phi_{(q, r \text { odd })}\right\}_{\mathrm{DB}}=i T_{(q, r \text { odd })}$

with

$$
\begin{aligned}
T_{(q, r \text { odd })}= & |x|^{2} p^{2}+2 i(\xi \cdot x)(\xi \cdot p) \\
& +2 r i^{n} q|x|^{r+1} \xi \xi^{\underline{n}[r-1]} F_{\underline{n}[r-1] \underline{m}} p^{\underline{m}} \\
& -2 r i^{n-1} q|x|^{r-1}(\xi \cdot x) \xi \underline{\underline{m}}[r] F_{\underline{m}[r]} \\
& +(-)^{\frac{r-1}{2}} r^{2} q^{2}|x|^{2 r} \xi \underline{\underline{m}}[r-1] F_{\underline{m}[r-1]^{\underline{k}}} \\
& \times F_{\underline{k n}[r-1]} \xi^{\underline{n}[r-1] \approx 0}
\end{aligned}
$$

being the world-line reparametrization generator in the presence of the interaction.

Similarly to the previously considered case of the interaction with the background electromagnetic field, one can write down the spinning particle's Hamiltonian

$\mathscr{H}_{(q, r \text { odd })}=\frac{\tilde{e}}{2} T_{(q, r \text { odd })}-a D-i \chi \Phi_{(q, r \text { odd })} \approx 0$

\footnotetext{
${ }^{5}$ We use the notation according to which a number in square brackets following an index stands for the set of antisymmetrized indices equal to that number.
} 
and the action functional

$S_{(q, r \text { odd })}=\int d \tau \mathscr{L}_{(q, r \text { odd }) \mathrm{ph}}$

where the Lagrangian expressed in terms of the phase-space variables has the form

$\mathscr{L}_{(q, r \text { odd }) \mathrm{ph}}=(p \cdot \dot{x})+\frac{i}{2}(\xi \cdot \dot{\xi})-\mathscr{H}_{(q, r \text { odd })}$

Integrating consecutively momentum $p^{\underline{m}}$ and dilatation gauge field $a$ yields two representations of the configurationspace Lagrangian:

$$
\begin{aligned}
\mathscr{L}_{(q, r \text { odd }) \text { conf }}= & \frac{1}{2 \tilde{e}|x|^{2}}(\dot{x}+a x)^{2}+\frac{i}{2}(\xi \cdot \dot{\xi}) \\
& -\frac{i}{|x|^{2}}(\xi \cdot x)(\xi \cdot \dot{x}) \\
& -r i^{n} q|x|^{r-1} \xi \underline{\underline{m}[r-1]} F_{\underline{m}[r-1] \underline{n}} \dot{x}^{\underline{n}} \\
& +\frac{i \chi}{\tilde{e}|x|}(\xi \cdot(\dot{x}+a x) \\
& \left.+(1-r) i^{n} q \tilde{e}|x|^{r+1} \xi^{\underline{m}[r]} F_{\underline{m}[r]}\right)
\end{aligned}
$$

and

$$
\begin{aligned}
\mathscr{L}_{(q, r \text { odd }) R P^{d}}= & \frac{1}{2 \tilde{e}|x|^{2}}(\dot{x} \theta \dot{x})+\frac{i}{2}(\xi \cdot \dot{\xi}) \\
- & \frac{i}{|x|^{2}}(\xi \cdot x)(\xi \cdot \dot{x}) \\
& -r i^{n} q|x|^{r-1} \xi^{\underline{m}[r-1]} F_{\underline{m}[r-1] \underline{n}} \dot{x}^{\underline{n}} \\
+ & \frac{i \chi}{\tilde{e}|x|}((\xi \theta \dot{x}) \\
& \left.+(1-r) i^{n} q \tilde{e}|x|^{r+1} \xi^{\underline{m}[r]} F_{\underline{m}[r]}\right) .
\end{aligned}
$$

In quantum theory the Hermitian operator associated with the odd constraint (40) is

$\Phi_{(q, r \text { odd }) \mathrm{H}}=|x|(\gamma \cdot p)+\frac{i(\gamma \cdot x)}{2|x|}+\frac{i^{n} q}{2^{\frac{r-1}{2}}}|x|^{r} \gamma^{\underline{m}[r]} F_{\underline{m}[r]} \approx 0$,

where the antisymmetrized product of $r \gamma$-matrices is defined as

$\gamma^{\underline{m}[r]}=\frac{1}{r !} \gamma^{\left[\underline{m}_{1}\right.} \gamma^{\underline{m}_{2}} \ldots \gamma^{\left.\underline{m}_{r}\right]}$.

Squaring the constraint (48) allows to obtain quantum version of the world-line supersymmetry algebra (41)

$\Phi_{(q, r \text { odd }) \mathrm{H}}^{2}=T_{(q, r \text { odd }) \mathrm{H}}$ and define the Hermitian operator corresponding to the generator of the world-line reparametrizations

$$
\begin{aligned}
T_{(q, r \text { odd }) \mathrm{H}}= & {[|x|(\gamma \cdot p)]^{2}+\frac{r i^{n} q}{2^{\frac{r-3}{2}}}|x|^{r+1} \gamma^{\underline{n}[r-1]} F_{\underline{n}[r-1]^{\underline{m}}} p_{\underline{m}} } \\
& +\frac{r i^{n+1} q}{2^{\frac{r-1}{2}}}|x|^{r-1}\left((\gamma \cdot x) \gamma^{\underline{m}[r]} F_{\underline{m}[r]}\right. \\
& \left.-|x|^{2} \gamma^{\underline{n}[r-1]} \partial^{\underline{m}} F_{\underline{m n}[r-1]}\right) \\
& +\frac{(-)^{n} q^{2}}{2^{r-1}}|x|^{2 r}\left(\gamma^{\underline{m}[r]} F_{\underline{m}[r]}\right)^{2}+i D_{H}-\frac{1}{4} \\
\approx & 0 .
\end{aligned}
$$

Let us note in passing that the square of $\gamma^{\underline{m}}{ }^{[r]} F_{\underline{m}[r]}$ can be expanded over the basis of the antisymmetrized products of $\gamma$-matrices using the relations given, e.g. in [57]

$$
\begin{aligned}
& \left(\gamma^{\underline{m}[r]} F_{\underline{m}[r]}\right)^{2}=\sum_{k=0}^{\frac{r-1}{2}}(-)^{k} \frac{(r !)^{2}}{(2 k+1) ![(r-2 k-1) !]^{2}}
\end{aligned}
$$

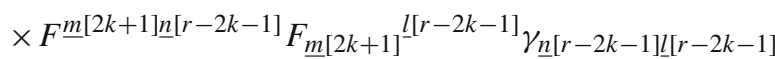

$$
\begin{aligned}
& =r^{2} F^{\underline{m n}[r-1]} F_{\underline{\underline{m}}}{ }^{l[r-1]} \gamma_{\underline{n}}[r-1] \underline{l}[r-1] \\
& -\frac{[r(r-1)]^{2}}{6} F^{\underline{m}[3] \underline{n}[r-3]} F_{\underline{m}[3]^{\underline{l}}[r-3]} \gamma_{\underline{n}[r-3] \underline{l}[r-3]} \\
& +\cdots+(-)^{\frac{r-1}{2}} r ! F^{\underline{m}}[r] F_{\underline{m}[r]} \text {. }
\end{aligned}
$$

Clearly which of the $(r+1) / 2$ terms actually contribute to the sum depends on the values of $r$ and the space-time dimension $d$.

Substitute now the realization (20) of the momentum as the differential operator to impose (48) and (51) on the configuration space wave function $\Psi(x)$. So we come to the first-order Dirac-type equation

$$
\begin{aligned}
\Phi_{(q, r \text { odd }) \mathrm{H}} \Psi(x)= & -i|x|(\gamma \cdot \partial) \Psi-\frac{i d(\gamma \cdot x)}{2|x|} \Psi \\
& +\frac{i^{n} q}{2^{\frac{r-1}{2}}}|x|^{r} \gamma^{\underline{m}[r]} F_{\underline{m}[r]} \Psi=0
\end{aligned}
$$

and the second-order Klein-Gordon-type equation

$$
\begin{aligned}
& T_{(q, r \text { odd }) \mathrm{H}} \Psi(x)=-[|x|(\gamma \cdot \partial)]^{2} \Psi \\
& -\frac{r i^{n+1} q}{2^{\frac{r-3}{2}}}|x|^{r+1} \gamma^{\underline{n}[r-1]} F_{\underline{n}[r-1]^{\underline{m}}} \partial_{\underline{m}} \Psi \\
& +\frac{r i^{n+1} q}{2^{\frac{r-1}{2}}}|x|^{r-1}\left((\gamma \cdot x) \gamma^{\underline{m}[r]} F_{\underline{m}[r]}\right. \\
& \left.-|x|^{2} \gamma^{\underline{n}[r-1]} \partial^{\underline{m}} F_{\underline{m n}[r-1]}\right) \Psi
\end{aligned}
$$




$$
\begin{aligned}
& +\frac{(-)^{n} q^{2}}{2^{r-1}}|x|^{2 r}\left(\gamma^{\underline{m}[r]} F_{\underline{m}[r]}\right)^{2} \Psi-\frac{d^{2}}{4} \Psi \\
= & 0 .
\end{aligned}
$$

For transformation of the above equations to the intrinsic coordinates let us first rewrite them in the inhomogeneous coordinates. Eq. (53) takes the form

$$
\begin{aligned}
\Phi_{(q, r \text { odd }) \mathrm{H}} \Psi(y)= & -i(\gamma \cdot \nabla) \Psi-\frac{i d}{2}(\gamma \cdot y) \Psi \\
& +\frac{i^{n} q}{2^{\frac{r-1}{2}}} \gamma^{\underline{m}[r]} F_{\underline{m}[r]} \Psi=0,
\end{aligned}
$$

where the $r$-form field strength in the homogeneous and inhomogeneous coordinates is related in the following way

$$
\begin{aligned}
|x|^{r} F_{\underline{m}[r]}(x) & =F_{\underline{m}[r]}(y), \quad F_{\underline{m}[r]}(y) \\
& =\left(\partial_{\left[\underline{m}_{1}\right.}+(r-1) y_{\left[\underline{m}_{1}\right.}\right) A_{\left.\underline{m}_{2} \ldots \underline{m}_{r}\right]}
\end{aligned}
$$

generalizing (25) and (26) for the electromagnetic field. The Klein-Gordon-type equation (54) in the inhomogeneous coordinates reads

$$
\begin{aligned}
T_{(q, r \text { odd }) \mathrm{H}} \Psi(y)= & -[(\gamma \cdot \nabla)]^{2} \Psi \\
& -\frac{r i^{n+1} q}{2^{\frac{r-3}{2}}} \gamma^{\underline{n}[r-1]} F_{\underline{n}[r-1]^{\underline{m}}} \nabla_{\underline{m}} \Psi \\
& +\frac{r i^{n+1} q}{2^{\frac{r-1}{2}}}\left((\gamma \cdot y) \gamma^{\underline{m}[r]} F_{\underline{m}[r]}\right. \\
& \left.-\gamma^{\underline{n}[r-1]} \nabla^{\underline{m}} F_{\underline{m n}[r-1]}\right) \Psi \\
& +\frac{(-)^{n} q^{2}}{2^{r-1}}\left(\gamma^{\underline{m}[r]} F_{\underline{m}[r]}\right)^{2} \Psi-\frac{d^{2}}{4} \Psi=0 .
\end{aligned}
$$

Then using the relations (27)-(32) we find wave equations describing gauge-invariant interaction of the spin $1 / 2$ field on the $A d S_{d}$ background with the odd-rank antisymmetric gauge field in the intrinsic coordinates

$$
\begin{aligned}
& i M^{-1}(\gamma \cdot y) \Phi_{(q, r \text { odd }) \mathrm{H}} M \psi(z) \\
& \quad=\rho^{m} D_{m} \psi+\frac{i^{n+1} q}{2^{\frac{r-1}{2}}} \rho^{m[r]} F_{m[r]} \psi=0
\end{aligned}
$$

and

$$
\begin{aligned}
- & M^{-1} T_{(q, r \text { odd }) \mathrm{H}} M \psi(z) \\
= & D^{2} \psi+\frac{r i^{n+1} q}{2^{\frac{r-3}{2}}} \rho^{m[r-1]} F_{m[r-1]^{n} D_{n} \psi} \\
& +\frac{r i^{n+1} q}{2^{\frac{r-1}{2}}} \rho^{m[r-1]} D^{n} F_{n m[r-1]} \psi \\
& -\frac{(-)^{n} q^{2}}{2^{r-1}}\left(\rho^{m[r]} F_{m[r]}\right)^{2} \psi+\frac{d(d-1)}{4} \psi=0 .
\end{aligned}
$$

The definition of the spinor covariant derivative coincides with (34) in the absence of electromagnetic field and the $r$-form field strength in the ambient-space and intrinsic coordinates is related as

$$
\begin{aligned}
F_{\underline{m}[r]}(y) & =\partial^{m_{1}} y_{\underline{m}_{1}} \ldots \partial^{m_{r}} y_{\underline{m}_{r}} F_{m[r]}(z), \\
F_{m[r]}(z) & =\partial_{\left[m_{1}\right.} A_{\left.m_{2} \ldots m_{r}\right]}(z) .
\end{aligned}
$$

\section{$3.2 r$ even}

In the case of $r$ even, the odd constraint takes the form

$$
\Phi_{(q, r \text { even })}=|x|(\xi \cdot p)+i^{n} q|x|^{r-1}(\xi \cdot x) \xi^{\underline{m}[r]} F_{\underline{m}[r]} \approx 0 .
$$

In this subsection $n=\frac{r}{2}-2\left[\frac{r}{4}\right]$. Similarly to the previously considered models, DB relations of this constraint with itself generate the world-line supersymmetry algebra

$$
\left\{\Phi_{(q, r \text { even })}, \Phi_{(q, r \text { even })}\right\}_{\mathrm{DB}}=i T_{(q, r \text { even })},
$$

where the world-line reparametrization generator equals

$$
\begin{aligned}
T_{(q, r \text { even })=} & |x|^{2} p^{2}+2 i(\xi \cdot x)(\xi \cdot p) \\
& +2 r i^{n} q|x|^{r}(\xi \cdot x) \xi^{\underline{n}[r-1]} F_{\underline{n}[r-1] \underline{m}} p^{\underline{m}} \\
& +2 i^{n} q|x|^{r} \xi^{\underline{m}[r]} F_{\underline{m}[r]}(x \cdot p) \\
& -(-)^{n} q^{2}|x|^{2 r}\left(\xi^{\underline{m}[r]} F_{\underline{m}[r]}\right)^{2} \approx 0 .
\end{aligned}
$$

The constraints (61), (63) and $D$ are the first-class constraints of the model and are used to define the spinning particle's Lagrangian and action functional

$$
\begin{aligned}
S_{(q, r \text { even })}=\int & d \tau \mathscr{L}_{(q, r \text { even }) \mathrm{ph}}: \\
\mathscr{L}_{(q, r \text { even }) \mathrm{ph}}= & (p \cdot \dot{x})+\frac{i}{2}(\xi \cdot \dot{\xi})-\frac{\tilde{e}}{2} T_{(q, r \text { even })} \\
& +a D+i \chi \Phi_{(q, r \text { even })} .
\end{aligned}
$$

Substituting explicit expressions for these constraints and integrating out the momentum allows to transfer from the phase-space to the configuration-space form of the Lagrangian

$$
\begin{aligned}
\mathscr{L}_{(q, r \text { even }) \text { conf }}= & \frac{1}{2 \tilde{e}|x|^{2}}(\dot{x}+a x)^{2}+\frac{i}{2}(\xi \cdot \dot{\xi}) \\
& -\frac{i}{|x|^{2}}(\xi \cdot x)(\xi \cdot \dot{x}) \\
& -r i^{n} q|x|^{r-2}(\xi \cdot x) \xi^{\underline{m}[r-1]} F_{\underline{m}[r-1] \underline{\underline{x}}} \dot{x}^{\underline{n}} \\
& +\frac{(-)^{n} q^{2}}{2} \tilde{e}|x|^{2 r}\left(\xi^{\underline{m}[r]} F_{\underline{m}[r]}\right)^{2}
\end{aligned}
$$




$$
\begin{aligned}
& +\frac{i \chi}{\tilde{e}|x|}(\xi \cdot(\dot{x}+a x) \\
& \left.+(1-r) i^{n} q \tilde{e}|x|^{r}(\xi \cdot x) \xi^{\underline{m}[r]} F_{\underline{m}[r]}\right) .
\end{aligned}
$$

Further integrating out the dilatation gauge field, one finds the Lagrangian that corresponds to the realization of the $A d S_{d}$ as a projective manifold with the degenerate metric

$$
\begin{aligned}
\mathscr{L}_{(q, r \text { even }) R P^{d}}= & \frac{1}{2 \tilde{e}|x|^{2}}(\dot{x} \theta \dot{x}) \\
& +\frac{i}{2}(\xi \cdot \dot{\xi})-\frac{i}{|x|^{2}}(\xi \cdot x)(\xi \cdot \dot{x}) \\
& -r i^{n} q|x|^{r-2}(\xi \cdot x) \xi^{\underline{m}[r-1]} F_{\underline{m}[r-1] \underline{n}} \dot{x}^{\underline{n}} \\
& +\frac{(-)^{n} q^{2}}{2} \tilde{e}|x|^{2 r}\left(\xi^{\underline{m}[r]} F_{\underline{m}[r]}\right)^{2} \\
& +\frac{i \chi}{\tilde{e}|x|}((\xi \theta \dot{x}) \\
& \left.+(1-r) i^{n} q \tilde{e}|x|^{r}(\xi \cdot x) \xi^{\underline{m}[r]} F_{\underline{m}[r]}\right) .
\end{aligned}
$$

Now we come to the discussion of the Dirac quantization of the model. Let us define the Hermitian operator associated with the odd constraint (61) as

$$
\begin{aligned}
\Phi_{(q, r \text { even }) \mathrm{H}}= & |x|(\gamma \cdot p)+\frac{i(\gamma \cdot x)}{2|x|} \\
& +\frac{i^{n} q}{2^{\frac{r}{2}}}|x|^{r-1}(\gamma \cdot x) \gamma^{\underline{m}[r]} F_{\underline{m}[r]} \approx 0 .
\end{aligned}
$$

As in the previous sections expression for the Hermitian operator that corresponds to the world-line reparametrization generator is obtained by requiring the closure of the world-line supersymmetry algebra

$$
\Phi_{(q, r \text { even }) \mathrm{H}}^{2}=T_{(q, r \text { even }) \mathrm{H}},
$$

where

$$
\begin{aligned}
& T_{(q, r \text { even }) \mathrm{H}}=[|x|(\gamma \cdot p)]^{2} \\
& +\frac{r i^{n} q}{2^{\frac{r}{2}-1}}|x|^{r}(\gamma \cdot x) \gamma^{[r-1]} F_{\underline{n}[r-1]^{\underline{m}}} p_{\underline{m}} \\
& +\frac{r i^{n+1} q}{2^{\frac{r}{2}}}|x|^{r}\left(\gamma^{\underline{m}[r]} F_{\underline{m}[r]}\right. \\
& \left.+(\gamma \cdot x) \gamma^{\underline{n}[r-1]} \partial^{\underline{m}} F_{\underline{m n}}[r-1]\right) \\
& -\frac{(-)^{n} q^{2}}{2^{r}}|x|^{2 r}\left(\gamma^{\underline{m}[r]} F_{\underline{m}[r]}\right)^{2}+i \\
& \left(1+\frac{i^{n-1} q}{2^{\frac{r}{2}-1}}|x|^{r} \gamma^{\underline{m}[r]} F_{\underline{m}[r]}\right) D_{\mathrm{H}}-\frac{1}{4} \approx 0 .
\end{aligned}
$$

Realizing momentum operator as the differential operator in configuration space allows to obtain Dirac-type and KleinGordon-type equations for the particle's wave function in the homogeneous coordinates

$$
\begin{aligned}
\Phi_{(q, r \text { even }) \mathrm{H}} \Psi(x)= & -i|x|(\gamma \cdot \partial) \Psi-\frac{i d(\gamma \cdot x)}{2|x|} \Psi \\
& +\frac{i^{n} q}{2^{\frac{r}{2}}}|x|^{r-1}(\gamma \cdot x) \gamma^{\underline{m}[r]} F_{\underline{m}[r]} \Psi=0
\end{aligned}
$$

and

$$
\begin{aligned}
T_{(q, r \text { even }) \mathrm{H}} \Psi(x)= & -[|x|(\gamma \cdot \partial)]^{2} \Psi \\
& -\frac{r i^{n+1} q}{2^{\frac{r}{2}-1}}|x|^{r}(\gamma \cdot x) \gamma^{\underline{n}[r-1]} F_{\underline{n}[r-1]^{\underline{m}}} \partial_{\underline{m}} \Psi \\
& +\frac{r i^{n+1} q}{2^{\frac{r}{2}}}|x|^{r}\left(\gamma^{\underline{\underline{m}}[r]} F_{\underline{m}[r]}\right. \\
& \left.+(\gamma \cdot x) \gamma^{\underline{n}[r-1]} \partial^{\underline{m}} F_{\underline{m n}[r-1]}\right) \Psi \\
& -\frac{(-)^{n} q^{2}}{2^{r}}|x|^{2 r}\left(\gamma^{\underline{\underline{m}}[r]} F_{\underline{m}[r]}\right)^{2} \Psi-\frac{d^{2}}{4} \Psi \\
& =0 .
\end{aligned}
$$

Note that $\Psi(x)$ is homogeneous of degree zero since $D_{\mathrm{H}} \Psi(x)=0$. In terms of the inhomogeneous coordinates $y^{\underline{m}}=|x|^{-1} x^{\underline{m}}$ these equations acquire the form

$$
\begin{aligned}
\Phi_{(q, r \text { even }) \mathrm{H}} \Psi(y)= & -i(\gamma \cdot \nabla) \Psi-\frac{i d}{2}(\gamma \cdot y) \Psi \\
& +\frac{i^{n} q}{2^{\frac{r}{2}}}(\gamma \cdot y) \gamma^{\underline{m}[r]} F_{\underline{m}[r]} \Psi=0
\end{aligned}
$$

and

$$
\begin{aligned}
T_{(q, r \text { even }) \mathrm{H}} \Psi(y)= & -[(\gamma \cdot \nabla)]^{2} \Psi \\
& -\frac{r i^{n+1} q}{2^{\frac{r}{2}-1}}(\gamma \cdot y) \gamma^{\underline{n}[r-1]} F_{\underline{n}[r-1]^{\underline{m}}} \nabla_{\underline{m}} \Psi \\
& +\frac{r i^{n+1} q}{2^{\frac{r}{2}}}\left(\gamma^{\underline{m}[r]} F_{\underline{m}[r]}\right. \\
& \left.+(\gamma \cdot y) \gamma^{\underline{n}[r-1]} \partial^{\underline{m}} F_{\underline{m n}[r-1]}\right) \Psi \\
& -\frac{(-)^{n} q^{2}}{2^{r}}\left(\gamma^{\underline{m}[r]} F_{\underline{m}[r]}\right)^{2} \Psi-\frac{d^{2}}{4} \Psi=0 .
\end{aligned}
$$

Connection between the $r$-form field strength in the homogeneous and inhomogeneous coordinates is given in (56). Using the transition relations (27)-(32) one can write the above equations in terms of the intrinsic coordinates. Eq. (73) transforms into the massless Dirac equation on $A d S_{d}$ coupled to external $r$-form field strength 


$$
\begin{aligned}
& i M^{-1}(\gamma \cdot y) \Phi_{(q, r \text { even }) \mathrm{H}} M \psi(z)=\rho^{m} D_{m} \psi \\
& +\frac{i^{n-1} q}{2^{\frac{r}{2}}} \rho^{m[r]} F_{m[r]} \psi=0 .
\end{aligned}
$$

The relation between the $r$-form field strength in the inhomogeneous and intrinsic coordinates is given in (60). Analogously the second-order equation (74) transforms into the generalization of the Klein-Gordon equation

$$
\begin{aligned}
- & M^{-1} T_{(q, r \text { even }) \mathrm{H}} M \psi(z) \\
= & D^{2} \psi+\frac{r i^{n+1} q}{2^{\frac{r}{2}-1}} \rho^{n[r-1]} F_{n[r-1]}{ }^{m} D_{m} \psi \\
& -\frac{r i^{n+1} q}{2^{\frac{r}{2}}} \rho^{n[r-1]} D^{m} F_{m n[r-1]} \psi \\
& +\frac{(-)^{n} q^{2}}{2^{r}}\left(\rho^{m[r]} F_{m[r]}\right)^{2} \psi \\
& +\frac{d(d-1)}{4} \psi=0 .
\end{aligned}
$$

\section{Conclusion}

In this note we have studied interactions with background electromagnetic or rank $(r-1)$ antisymmetric gauge fields of the minimally-supersymmetric massless spinning particle in anti-de Sitter space-time. $d$-dimensional anti-de Sitter space-time has been realized as a real projective manifold parametrized by the homogeneous coordinates. For all of the considered interactions we have found the set of three first-class constraints, one odd and two even, that generate extended world-line supersymmetry algebra. The constraints are the classical generators of $1 d$ supersymmetry, reparametrizations and rescalings of the space-time homogeneous coordinates. Various forms of the spinning particle's Lagrangian both in terms of the phase-space and configuration-space variables have been derived. Then the quantum realization of the classical constraint algebra by the Hermitian operators has been found. The form of the Hermitian operator associated with the classical generator of the world-line reparametrizations is unambiguously fixed by the closure of the quantum algebra of the constraints. The realization of the Hermitian momentum operator as the differential operator in configuration space yields first- and secondorder equations for the particle's wave function in the presence of background electromagnetic field or antisymmetric gauge fields. These equations have been presented both in the homogeneous and inhomogeneous coordinates of the ambient space. Finally using known transition relations between the ambient and intrinsic coordinates they have been written in the conventional form of extended Dirac and Klein-Gordon equations in $A d S_{d}$.
Let us note that although we treated independently interactions of the spinning particle with electromagnetic and $(r-1)$-form gauge fields, along the same lines it is possible to consider simultaneous coupling to a number of gauge fields and electromagnetic field. One can also consider interactions with mixed symmetry fields that carry even number of indices in each set of the antisymmetrized indices.

The part of our discussion that concerned transition of the equations for particle's wave function from the ambient-space to intrinsic coordinates assumed implicitly that $\operatorname{Spin}(1, d-1)$ and $\operatorname{Spin}(2, d-1)$ spinor representations have equal dimension that is the case for $d$ even. So one of possible generalizations is to consider the case of $d$ odd that presumably requires introduction of additional (odd) variables and constraints to impose chirality projection on the spinor wave function in $d+1$ dimensions.

As a further development of the results reported here it is possible to consider interaction of the spinning particle with the Yang-Mills field, to look for the superfield formulation and to describe particles with other values of spin.

Data Availability Statement This manuscript has no associated data or the data will not be deposited. [Authors' comment: There are no extra data associated with this article other than the article itself.]

Open Access This article is distributed under the terms of the Creative Commons Attribution 4.0 International License (http://creativecomm ons.org/licenses/by/4.0/), which permits unrestricted use, distribution, and reproduction in any medium, provided you give appropriate credit to the original author(s) and the source, provide a link to the Creative Commons license, and indicate if changes were made. Funded by SCOAP $^{3}$.

\section{References}

1. A. Barducci, R. Casalbuoni, L. Lusanna, Supersymmetries and the pseudoclassical relativistic electron. Nuovo Cim. A 35, 377 (1976)

2. L. Brink, S. Deser, B. Zumino, P. Di Vecchia, P.S. Howe, Local supersymmetry for spinning particles. Phys. Lett. B 64, 435 (1976) Erratum: Phys. Lett. B 68 (1977) 488

3. F.A. Berezin and M.S. Marinov, Classical spin and Grassmann algerba, JETP Lett. 21 (1975) 320 [Pisma v ZhETF 21 (1975) 678]

4. F.A. Berezin, M.S. Marinov, Particle spin dynamics as the grassmann variant of classical mechanics. Ann. Phys. 104, 336 (1977)

5. L. Brink, P. Di Vecchia, P.S. Howe, A Lagrangian formulation of the classical and quantum dynamics of spinning particles. Nucl. Phys. B 118, 76 (1977)

6. A. Barducci, Pseudoclassical description of relativistic spinning particles with anomalous magnetic moment. Phys. Lett. B 118, 112 (1982)

7. A.A. Zheltukhin, Superfield description of a particle with spin and anomalous magnetic moment, Theor. Math. Phys. 65 (1985) 1072 [Teor. Mat. Fiz. 65 (1985) 151]

8. A.A. Zheltukhin, The Kaluza-Klein mechanism and a superfield description of spinning particles interactions. Phys. Lett. B 168, 43 (1986)

9. A. Barducci, R. Casalbuoni, L. Lusanna, Classical scalar and spinning particles interacting with external Yang-Mills fields. Nucl. Phys. B 124, 93 (1977) 
10. A.P. Balachandran, P. Salomonson, B.S. Skagerstam, J.O. Winnberg, Classical description of particle interacting with nonabelian gauge field. Phys. Rev. D 15, 2308 (1977)

11. A. Barducci, R. Casalbuoni, L. Lusanna, Classical spinning particles interacting with external gravitational fields. Nucl. Phys. B 124, 521 (1977)

12. A.A. Zheltukhin, On superfield structure of action for supersymmetric particle, Sov. J. Nucl. Phys. 46 (1987) 1072 [Yad. Fiz. 46 (1987) 1791]

13. P.S. Howe, S. Penati, M. Pernici, P.K. Townsend, Wave equations for arbitrary spin from quantization of the extended supersymmetric spinning particle. Phys. Lett. B 215, 555 (1988)

14. S.M. Kuzenko, Z.V. Yarevskaya, Conformal invariance, $N$ extended supersymmetry and massless spinning particles in anti-de Sitter space. Mod. Phys. Lett. A 11, 1653 (1996). arXiv:hep-th/9512115

15. R. Marnelius, B.E.W. Nilsson, Equivalence between a massive spinning particle in Minkowski space and a massless one in a de Sitter space. Phys. Rev. D 20, 839 (1979)

16. R. Marnelius, Manifestly conformally covariant description of spinning and charged particles. Phys. Rev. D 20, 2091 (1979)

17. I. Bars, C. Deliduman, Gauge symmetry in phase space with spin: a basis for conformal symmetry and duality among many interactions. Phys. Rev. D 58, 106004 (1998). arXiv:hep-th/9806085

18. I. Bars, Two time physics with gravitational and gauge field backgrounds. Phys. Rev. D 62, 085015 (2000). arXiv:hep-th/0002140

19. P.A.M. Dirac, The electron wave equation in de Sitter space. Ann. Math. 36, 657 (1935)

20. P.A.M. Dirac, Wave equations in conformal space. Ann. Math. 37, 429 (1936)

21. G. Mack, A. Salam, Finite component field representations of the conformal group. Ann. Phys. 53, 174 (1969)

22. S. Ferrara, A.F. Grillo, R. Gatto, Tensor representations of conformal algebra and conformally covariant operator product expansion. Ann. Phys. 76, 161 (1973)

23. S. Ferrara, R. Gatto, A.F. Grillo, Conformal algebra in space-time and operator product expansion. Springer Tracts Mod. Phys. 67, 1 (1973)

24. C. Fronsdal, Singletons and massless, integral-spin fields on de Sitter space. Phys. Rev. D 20, 848 (1979)

25. J. Fang, C. Fronsdal, Massless, half-integer-spin fields in de Sitter space. Phys. Rev. D 22, 1361 (1980)

26. L. Cornalba, M.S. Costa, J. Penedones, Deep inelastic scattering in conformal QCD. JHEP 1003, 133 (2010). arXiv:0911.0043 [hepth]

27. S. Weinberg, Six-dimensional methods for four-dimensional conformal field theories. Phys. Rev. D 82, 045031 (2010). arXiv:1006.3480 [hep-th]

28. J. Penedones, Writing CFT correlation functions as AdS scattering amplitudes. JHEP 1103, 025 (2011). arXiv:1011.1485 [hep-th]

29. X. Bekaert, M. Grigoriev, Notes on the ambient approach to boundary values of $A d S$ gauge fields. J. Phys. A: Math. Theor. 46, 214008 (2013). arXiv:1207.3439 [hep-th]

30. V.E. Didenko, E.D. Skvortsov, Towards higher-spin holography in ambient space of any dimension. J. Phys. A: Math. Theor. 46, 214010 (2013). arXiv:1207.6786 [hep-th]

31. C. Sleight, Metric-like methods higher spin holography. PoS Modave 2016, 003 (2017). arXiv:1701.08360 [hep-th]

32. T. Adamo, D. Skinner, J. Williams, Twistor methods for Ad $S_{5}$. JHEP 1608, 167 (2016). arXiv:1607.03763 [hep-th]

33. A.S. Arvanitakis, A.E. Barns-Graham, P.K. Townsend, Twistor description of spinning particles in AdS. JHEP 1801, 059 (2018). arXiv:1710.09557 [hep-th]

34. A.S. Arvanitakis, A.E. Barns-Graham, P.K. Townsend, $A d S$ particles and manifest (super)isometries. Phys. Rev. Lett. 118, 141601 (2017). arXiv:1608.04380 [hep-th]
35. P. Claus, J. Rahmfeld, Y. Zunger, A simple particle action from a twistor parametrization of $A d S_{5}$. Phys. Lett. B 466, 181 (1999). arXiv:hep-th/9906118

36. D.V. Uvarov, Supertwistor formulation for massless superparticle in $A d S_{5} \times S^{5}$ superspace. Nucl. Phys. B 936, 690 (2018). arXiv: 1807.08318 [hep-th]

37. A. Ferber, Supertwistors and conformal supersymmetry. Nucl. Phys. B 132, 55 (1978)

38. I. Bars, Twistors and 2T-physics. AIP Conf. Proc. 767, 3 (2005). arXiv:hep-th/0502065

39. I. Bars, Lectures on twistors, arXiv:hep-th/0601091

40. D.V. Uvarov, A.A. Zheltukhin, Hamiltonian structure and noncommutativity in $p$-brane models with exotic supersymmetry. JHEP 0403, 063 (2004). arXiv:hep-th/0310284

41. G. Bonelli, On the covariant quantization of tensionless bosonic strings in AdS spacetime. JHEP 0311, 028 (2003). arXiv:hep-th/0309222

42. L.A. Cabral, V.O. Rivelles, Particles and strings in degenerate metric spaces. Class. Quantum Grav. 17, 1577 (2000). arXiv:hep-th/9910163

43. R.R. Metsaev, Massless mixed symmetry bosonic free fields in $d$ dimensional anti-de Sitter space-time. Phys. Lett. B 354, 78 (1995)

44. R.R. Metsaev, Arbitrary spin massless bosonic fields in $d$ dimensional anti-de Sitter space. Lect. Notes Phys. 524, 331 (1999). arXiv:hep-th/9810231

45. R.R. Metsaev, Fermionic fields in the $d$-dimensional anti-de Sitter spacetime. Phys. Lett. B 419, 49 (1998). arXiv:hep-th/9802097

46. D.V. Uvarov, Massless spinning particle and null-string on $A d S_{d}$ : projective-space approach. J. Phys. A: Math. Theor. 51, 285402 (2018). arXiv:1707.05761 [hep-th]

47. L. Lusanna, B. Milewski, $N=2$ super-Yang-Mills and supergravity constraints from coupling to a supersymmetric particle. Nucl. Phys. B 247, 396 (1984)

48. M. Rocek, W. Siegel, P. van Nieuwenhuizen, A.E. van de Ven, Covariant superparticle quantization in a supermaxwell background. Phys. Lett. B 227, 87 (1989)

49. F. Delduc, E. Sokatchev, Superparticle with extended worldline supersymmetry. Class. Quantum Grav. 9, 361 (1992)

50. I.A. Bandos, AYu. Nurmagambetov, Generalized action principle and extrinsic geometry for $N=1$ superparticles. Class. Quantum Grav. 14, 1597 (1997). arXiv:hep-th/9610098

51. D.V. Uvarov, A.A. Zheltukhin, $N=2$ massive superparticle: the minimality principle and $\kappa$-symmetry. Phys. Rev. D 61, 015004 (2000). arXiv:hep-th/9901067

52. A.A. Zheltukhin, D.V. Uvarov, $\kappa$-symmetry and anomalous magnetic moment of superparticles. JETP Lett. 67, 888 (1998)

53. P.S. Howe, S. Penati, M. Pernici, P.K. Townsend, A particle mechanics description of antisymmetric tensor fields. Class. Quantum Grav. 6, 1125 (1989)

54. A.C. Davis, A.J. Macfarlane, P.C. Popat, J.W. van Holten, The quantum mechanics of the supersymmetric nonlinear $\sigma$-model. J. Phys. A 17, 2945 (1984)

55. A.J. Macfarlane, P.C. Popat, The quantum mechanics of the $N=2$ extended supersymmetric nonlinear $\sigma$-model. J. Phys. A 17, 2955 (1984)

56. A.V. Smilga, How to quantize supersymmetric theories. Nucl. Phys. B 292, 363 (1987)

57. W. Mueck, General (anti)commutators of gamma matrices, arXiv:0711.1436 [hep-th] 